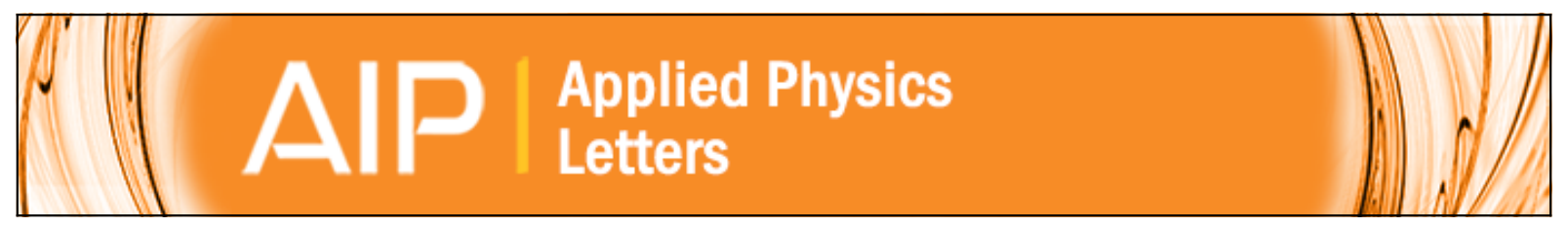

\title{
Electroluminescence in polymer-fullerene photovoltaic cells
}

Heejoo Kim, Jin Young Kim, Sung Heum Park, Kwanghee Lee, Youngeup Jin, Jinwoo Kim, and Hongsuk Suh

Citation: Applied Physics Letters 86, 183502 (2005); doi: 10.1063/1.1924869

View online: http://dx.doi.org/10.1063/1.1924869

View Table of Contents: http://scitation.aip.org/content/aip/journal/apl/86/18?ver=pdfcov

Published by the AIP Publishing

\section{Articles you may be interested in}

Charge-transfer states in conjugated polymer/fullerene blends: Below-gap weakly bound excitons for polymer photovoltaics

Appl. Phys. Lett. 93, 053307 (2008); 10.1063/1.2969295

Trilayer hybrid polymer-quantum dot light-emitting diodes

Appl. Phys. Lett. 84, 2925 (2004); 10.1063/1.1699476

Creation of a gradient polymer-fullerene interface in photovoltaic devices by thermally controlled interdiffusion Appl. Phys. Lett. 81, 4607 (2002); 10.1063/1.1522830

Transient optical studies of charge recombination dynamics in a polymer/fullerene composite at room temperature

Appl. Phys. Lett. 81, 3001 (2002); 10.1063/1.1512943

Fluorene-fluorenone copolymer: Stable and efficient yellow-emitting material for electroluminescent devices J. Appl. Phys. 92, 3495 (2002); 10.1063/1.1502920

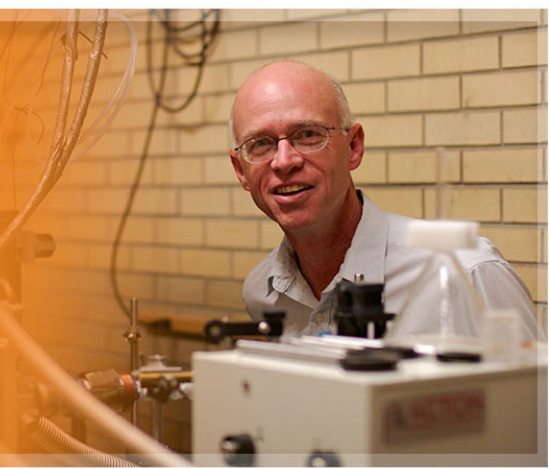




\title{
Electroluminescence in polymer-fullerene photovoltaic cells
}

\author{
Heejoo Kim, Jin Young Kim, Sung Heum Park, and Kwanghee Lee ${ }^{a}$ \\ Department of Physics, Pusan National University, Pusan 609-735, South Korea \\ Youngeup Jin, Jinwoo Kim, and Hongsuk Suh \\ Department of Chemistry, Pusan National University, Pusan 609-735, South Korea
}

(Received 20 December 2004; accepted 22 March 2005; published online 28 April 2005)

\begin{abstract}
We report electroluminescence (EL) in photovoltaic (PV) cells based on semiconducting polymer-fullerene composites. By applying a forward bias to the PV cells, the devices exhibited a clear EL action with a peak around $1.5 \mathrm{eV}$. We ascribe this peak to an "electric field-assisted exciplex" formed between the electrons in the fullerenes and the holes in the polymers, thereby resulting in radiative recombination in the composites. This finding is totally unexpected because of a strong photoluminescence quenching in the same materials. Since the same devices also showed typical photovoltaic effects under illumination, our results demonstrate a dual functionality in one device; polymer photovoltaic cells and polymer light-emitting diodes. (C) 2005 American Institute of Physics. [DOI: 10.1063/1.1924869]
\end{abstract}

Semiconducting polymer-fullerene $\left(\mathrm{C}_{60}\right)$ composites continue to be promising materials for organic photovoltaics. ${ }^{1}$ Recent progress in these polymer photovoltaics results in power conversion efficiencies around $3 \%-4 \%$ under AM1.5 (AM=air mass) irradiation. ${ }^{2,3}$ This class of devices is based on an interesting photophysical phenomenon, called "ultrafast photoinduced charge transfer." After photoexcitation of the semiconducting polymers, the excited electrons in the polymers transfer to $\mathrm{C}_{60}$ within $\sim 50 \mathrm{fs}$ timescale. ${ }^{4}$ Since this process is faster than any other competing radiative and nonradiative decay processes, significant photoluminescence (PL) quenching occurs in the composites. ${ }^{4}$ The inhibition of early time recombination offers a way to efficient charge separation, which stimulates the application of high-efficiency photodiodes and photovoltaic (PV) cells using these composites.

It is reported that the back transfer of the electrons from $\mathrm{C}_{60}$ to the polymer is remarkably slow in the order of miliseconds. ${ }^{5,6}$ Steady-state photoinduced absorption (PIA) and nearly steady-state PIA experiments have confirmed that these transferred electrons are relaxed in the lowest unoccupied molecular orbital (LUMO) of $\mathrm{C}_{60}{ }^{6-8}$ Previous PL spectroscopic studies suggest that the recombination between the LUMO of $\mathrm{C}_{60}$ and the $\pi$ band of the polymers is a nonradiative decay based on an absence of the PL signature in the corresponding energy ranges. ${ }^{6,7}$ This nonradiative nature originates from the lattice relaxation of the transferred electrons in $\mathrm{C}_{60}$ with a Jahn-Teller-type distortion. ${ }^{9,10}$ The relaxation process induces an interfacial barrier at the interfaces between the semiconducting polymers and $\mathrm{C}_{60}$, thereby forming metastable states in $\mathrm{C}_{60}$ with $\sim \mu$ s lifetime. ${ }^{8}$ However, the interfacial barriers arising from such an electronlattice coupling can be overcome by applying an additional external force such as electric field or by thermal activation. In such a case, it might be possible to induce radiative black recombination in these systems by applying substantial electric field. In particular, since relatively strong electric field can be applied in recent polymer devices, the possibility of electroluminescence (EL) in polymer-fullerene devices is

\footnotetext{
${ }^{a)}$ Electronic mail: kwhlee@pusan.ac.kr
}

quite plausible. In this work, we report a clear EL action in the polymer-fullerene PV cells under forward bias above $5 \mathrm{~V}$. Composite solutions of poly-[2-methoxy-5-(2'-ethylhexyloxy)-1,4-phenylene vinylene] (MEH-PPV), and soluble $\mathrm{C}_{60},\{6\}-1-[3-($ methoxycarbonyl)propyl]-\{5\}-1-phenyl [5,6]$\mathrm{C}_{61}$ (PCBM) were prepared by blending parent solutions (MEH-PPV in tetrahydrofuran and PCBM in chlorobenzene) with various weight percents (wt \%) of PCBM; 0, 5, 10, 20, and $50 \mathrm{wt} \%$. These composite solutions were stirred at room temperature for $12 \mathrm{~h}$. Each solution was spun on precleaned UV-graded fused silica substrates for optical absorption and PL spectra measurements. All of the cast films did not show any phase segregation of MEH-PPV and PCBM. Optical absorption spectra were recorded by a Varian 5E UV-VIS-NIR spectrophotometer, while Oriel Instaspec IV charge-coupled device detection system in combination with a solid state laser $(532 \mathrm{~nm})$ was used for the PL spectra measurements.

Polymer-fullerene PV cells of various concentrations of $\operatorname{PCBM}(0,5,10,20$, and $50 \mathrm{wt} \%)$ were fabricated with a typical device structure of ITO/PEDOT:PSS/MEH-PPV + PCBM composite/Al. Poly(3,4-ethylenedioxylene thiphene)-polystyrene sulphonic acid (PEDOT:PSS) was used as a buffer layer, which acts as a hole collecting layer and also decreases morphological roughness of the ITO surfaces. The thicknesses of the composite films were around $100 \mathrm{~nm}$. Then, an aluminum (Al) electrode was deposited by using a thermal evaporation in a vacuum of about $5 \times 10^{-5}$. The current density-voltage $(J-V)$ characteristics of the devices were measured under illumination and in the dark using a Keithley 236 source measure unit (SMU). A solid state laser was used as a monochromatic light source $(532 \mathrm{~nm}$, $30 \mathrm{~mW} / \mathrm{cm}^{2}$ ) for the photovoltaic measurements. The luminescence-voltage $(L-V)$ characteristics of these devices were obtained by a Keithley 236 SMU equipped with a calibrated photomultiplier tube.

PL quenching in donor-acceptor composites is a useful indication for the efficient charge transfer between the two components. ${ }^{4}$ Figure 1(a) shows the PL spectra of the MEHPPV/PCBM composite films with various concentrations of PCBM. The results exhibit a significant PL quenching of the MEH-PPV emission in the composites. Even for the lowest 

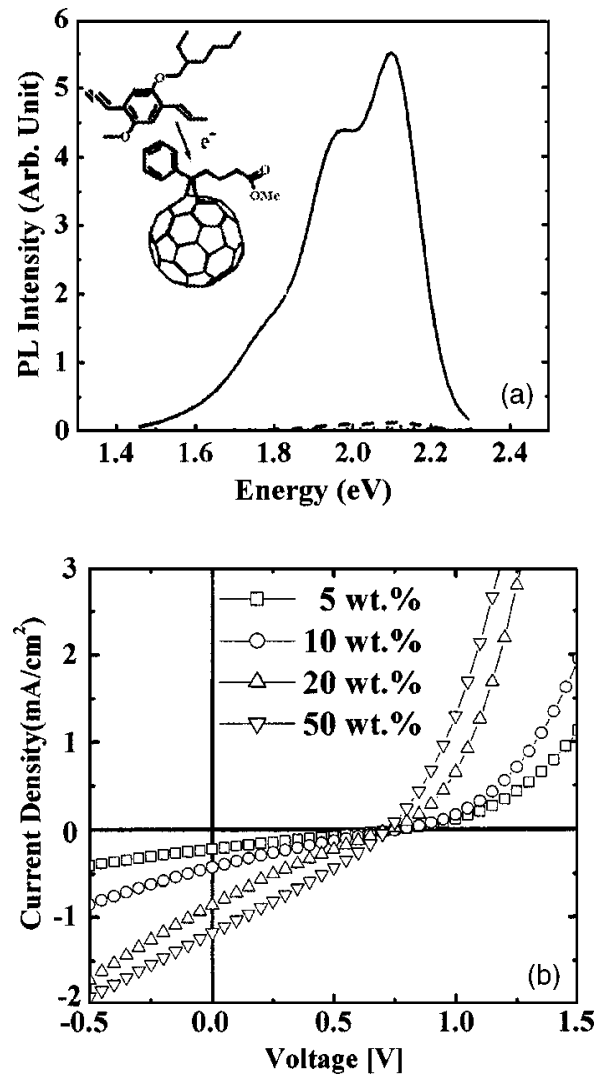

FIG. 1. (a) PL spectra of the MEH-PPV+PCBM composite films with various PCBM concentrations. The inset shows the photoinduced charge transfer between MEH-PPV and PCBM. (b) $J-V$ characteristics of the ITO/PEDOT:PSS/MEH-PPV +PCBM/Al device under $532 \mathrm{~nm}$ illumination.

PCBM concentration $(\sim 5 \%)$ film, the PL is completely quenched with almost no spectral feature over the entire ranges. This observation obviously indicates that the charge transfer from MEH-PPV to PCBM occurs efficiently, and the back-transfer proceeds with the nonradiative recombination process. ${ }^{11,12}$

Utilizing those composites as an active layer, we fabricated typical PV cells with a structure of ITO/PEDOT:PSS/MEH-PPV+PCBM/Al. Figure 1(b) shows the $J-V$ curves of the PV cells under monochromatic illumination $\left(\lambda=532 \mathrm{~nm}, 30 \mathrm{~mW} / \mathrm{cm}^{2}\right)$. The data clearly indicate typical PV effects for all devices. The short circuit current $\left(I_{\mathrm{sc}}\right)$ increases gradually with increasing PCBM concentration, while the open circuit voltage $\left(V_{\mathrm{oc}}\right)$ remains around $0.8 \mathrm{~V}$ for all devices. From these PV parameters, we evaluate the power conversion efficiencies $\left(\eta_{p}\right)$ as $\eta_{p}$ $=0.13 \%-0.8 \%$ for these devices. Using a higher PCBM ratio $(\sim 75$ wt $\%)$ composite, we achieved $\eta_{p}=2.3 \%$ (not shown here) under monochromatic light excitation. ${ }^{13}$

Using the same devices as above, we further applied the forward bias up to $\sim 10 \mathrm{~V}$ without any illumination. Then, we surprisingly observed that these PV cells exhibit a typical EL under forward bias as shown in Fig. 2. The light emission starts above a turn voltage around $5 \mathrm{~V}$ and reaches the value above $\sim 20 \mathrm{~cd} / \mathrm{m}^{2}$ at $10 \mathrm{~V}$ for the lowest concentration device (5\% device). The luminous intensity decreases with increasing PCBM concentration. Although the luminance of the composite devices is weak as compared with that of the typical polymer light-emitting diodes (P-LED), this observa-

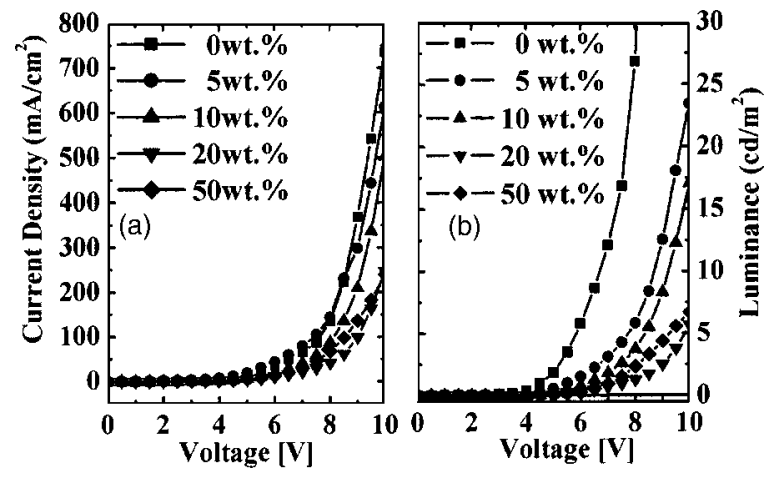

FIG. 2. Current density-voltage and luminance-voltage characteristics of the typical ITO/PEDOT: PSS/MEH-PPV + PCBM/Al devices.

tion is quite substantial. We precisely duplicated the experiments with consistent results both in the PV responses and EL actions in this system. Moreover, we also observed identical effects using other electroluminescent polymer systems, such as composites of poly [2-methoxy-5-( $3^{\prime}, 7^{\prime}$-dimethyloctyloxy)-p-phenylene vinylene $]\left(\mathrm{OC}_{1} \mathrm{OC}_{10}-\mathrm{PPV}\right)$ and PCBM, and composites of polyalkyfluorene derivative with $\mathrm{C}_{60}$ pendant. ${ }^{14}$ Therefore, we can safely conclude that this is a general phenomenon for those systems of electroluminescent polymer-fullerene composites.

Considering such a complete PL quenching in the same materials [see Fig. 1(a)] even for the lowest concentration of PCBM (5\%), this observation is somewhat unexpected. One might consider that some nonideal states of the active layers, such as phase segregation in the composites, would be responsible for this observation. However, obvious function as photovoltaic cells for the same devices clearly rules out such a possibility [see Fig. 1(b)]. In general, the failure of the formation of the interpenetrating networks in the composites would lead to a poor photovoltaic performance.

In order to clarify the origin of light emission in the composite PV cells, therefore, we have measured the EL spectra of the devices as shown in Fig. 3(a). The low concentration devices (5 and $10 \mathrm{wt} \%$ ) show more or less similar EL spectra with that of the pure MEH-PPV (0\%) with a peak around $2.1 \mathrm{eV}$, which corresponds to the characteristic luminescent peak of MEH-PPV, and with a weak feature around $1.7 \mathrm{eV}$. However, the device of the $20 \mathrm{wt} \%$ PCBM exhibits a dramatic change in the spectrum; the $1.7 \mathrm{eV}$ feature grows to the prominent peak and a new peak develops around 1.5 $\mathrm{eV}$. For the $50 \mathrm{wt} \%$ device, the $1.5 \mathrm{eV}$ peak dominates the spectrum with completely suppressed features of the $2.1 \mathrm{eV}$ peak. Although previous PL studies on the $\mathrm{C}_{60}$ thin films unambiguously assign the $1.7 \mathrm{eV}$ peak to the emission from $\mathrm{PCBM},{ }^{15}$ the origin of the $1.5 \mathrm{eV}$ peak is unclear.

As shown in the energy level diagram of the devices in Fig. 3(b), three kinds of recombination are possible in their excited states; transitions I, II, and III. Transition I corresponds to the $\pi-\pi^{*}$ recombination of MEH-PPV $(2.1 \mathrm{eV})$, while the highest occupied molecular orbital (HOMO)LUMO transition of PCBM (transition II) yield the $1.7 \mathrm{eV}$ peak. Transition III is a direct recombination between the two components, and generally characterized as an "exciplex." ${ }^{16}$ We attribute this to the $1.5 \mathrm{eV}$ feature.

Although we can easily assign those features in the EL spectra as above, the questions arise how the EL spectra evolve with the concentration of PCBM in the composites, 

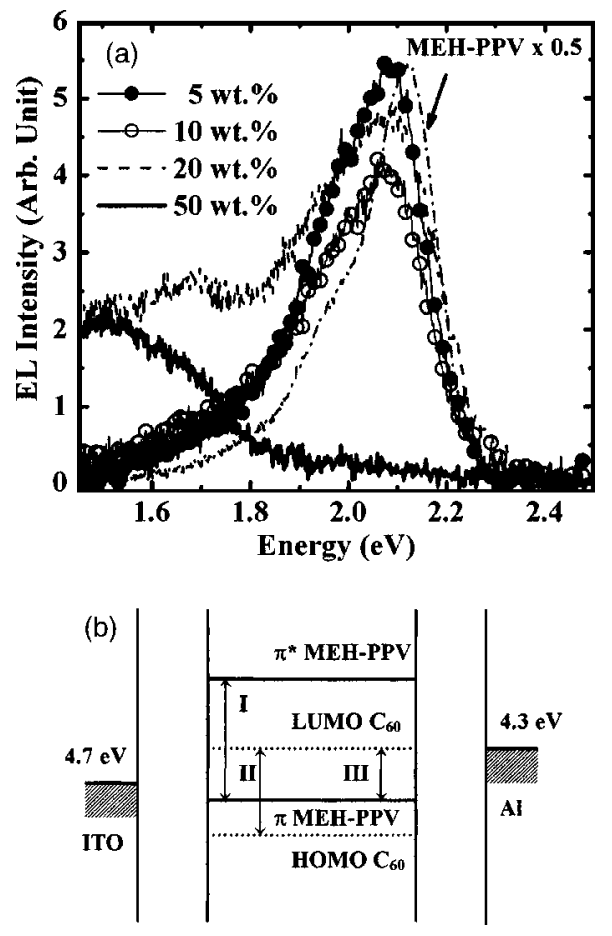

FIG. 3. (a) EL spectra of the devices using the MEH-PPV + PCBM composites with a sandwich structure of ITO/PEDOT:PSS/MEH-PPV $+\mathrm{PCBM} / \mathrm{Al}$. (b) Energy level diagram of the MEH-PPV $+\mathrm{C}_{60}$ composites. I: $\pi-\pi^{*}$ transition in MEH-PPV; II: HOMO-LUMO transition in $\mathrm{C}_{60}$; and III: direct transition between the electrons in $\mathrm{C}_{60}$ and holes in MEH-PPV.

and why transition III appears to be radiative in the EL spectra in contrast with its nonradiative nature in the PL spectra. The first question can be understood as follows. In such a bulk heterojunction photovoltaic geometry, charge carriers can be injected into both components, MEH-PPV and PCBM. However, since the percolation threshold in this system is around $20 \mathrm{wt} \%$ for PCBM,${ }^{17}$ the low concentration devices (5 and $10 \mathrm{wt} \%)$ are dominated by the charges injected mainly into the MEH-PPV, thereby yielding only the characteristic $2.1 \mathrm{eV}$ peak of MEH-PPV. For the $20 \mathrm{wt} \%$ device, the PCBM networks start to form and show the bulk nature of PCBM. ${ }^{18}$ In such a case, the charge carriers are injected into both components and induce transitions in each components; transition I at $2.1 \mathrm{eV}$ for MEH-PPV, and transition II at $1.7 \mathrm{eV}$ for PCBM. Since most of the LUMO levels of PCBM might be occupied by directly injected electrons from the $\mathrm{Al}$ electrode, the charge transfer from MEHPPV to $\mathrm{C}_{60}$ would be unfavorable for the $20 \mathrm{wt} \%$ device. We believe this would be also true even for the low concentration devices (5 and $10 \mathrm{wt} \%$ ). Although the low concentration devices are below the percolation threshold for PCBM, substantial portion of the PCBM might be occupied by the directly injected charges from the electrode, thereby prohibiting electron transfer from MEH-PPV into $\mathrm{C}_{60}$. This might be the reason why the $2.1 \mathrm{eV}$ peak is still dominant in the EL spectra of the low concentration devices in contrast with its complete quenching in the corresponding PL spectra.

When the concentration of PCBM increases to $50 \mathrm{wt} \%$, the network of PCBM would be uniformly distributed over the MEH-PPV matrix with a closer distance for the exciton dissociation. ${ }^{18}$ In such a situation, the injected electrons in MEH-PPV transfer to the LUMO levels of PCBM, and the holes injected to the PCBM part move to the HOMO of MEH-PPV. In particular, when a strong electric field is applied over thin active layers, the charges form an exciplex by reducing the interfacial barrier at the heterojunction as similar to the case of the bilayer-type organic-LEDs. ${ }^{19,20}$ These exciplex recombine eventually with a light emission, corresponding to the $1.5 \mathrm{eV}$ feature.

In conclusion, a dual functionality in one device is demonstrated by observing an EL action in the polymer-fullerene photovoltaic cells under forward bias. Moreover, the EL spectra show a systematic evolvement of a peak around 1.5 $\mathrm{eV}$ with increasing fullerene concentration in the composites. We ascribe this peak to an "electric field-assisted exciplex" formed between the electrons in the fullerenes and the holes in the polymers. The strong electric field in such thin-film devices reduces the barriers of the meta-stable electrons in $\mathrm{C}_{60}$, thereby inducing radiative recombination in the polymer-fullerene composites. We expect that such a dual functionality will provide an opportunity to create a smart display equipped with a self-energy supplying capability.

This work was supported by the MIC (Ministry of Information \& Communication), Korea, under the ITRC (Information Technology Research Center) support program supervised by the IITA (Institute of Information Technology Assessment).

${ }^{1}$ C. J. Brabec, N. S. Sariciftci, and J. C. Hummelen, Adv. Funct. Mater. 11, 15 (2001).

${ }^{2}$ S. E. Shaheen, C. J. Brabec, N. S. Sariciftci, F. Padinger, T. Fromherz, and J. C. Hummelen, Appl. Phys. Lett. 78, 841 (2001).

${ }^{3}$ I. Riedel and V. Dyakonov, Phys. Status Solidi A 201, 1332 (2004).

${ }^{4}$ N. S. Sariciftci, L. Smilowitz, A. J. Heeger, and F. Wudl, Science 258, 1474 (1992).

${ }^{5}$ C. J. Brabec, G. Zerza, G. Cerllo, S. DeSilvestri, S. Luzatti, J. C. Hummelen, and N. S. Sariciftci, Chem. Phys. Lett. 340, 232 (2001).

${ }^{6}$ L. Smilowitz, N. S. Sariciftci, R. Wu, C. Gettinger, A. J. Heeger, and F. Wudl, Phys. Rev. B 47, 13835 (1993).

${ }^{7}$ N. S. Sariciftci and A. J. Heeger, in Handbook of Organic Conductive Molecules and Polymers, edited by H. S. Nalwa (Wiley, New York, 1996), Vol. 1.

${ }^{8}$ K. Lee, R. A. J. Janssen, N. S. Sariciftci, and A. J. Heeger, Phys. Rev. B 49, 5781 (1994).

${ }^{9}$ V. Dyakonov, G. Zoriniants, M. Scharger, C. J. Brabec, R. A. J. Janssen, J. C. Hummelen, and N. S. Sariciftci, Phys. Rev. B 59, 8019 (1999).

${ }^{10}$ M. A. Greaney and S. M. Gorun, J. Phys. Chem. 95, 7142 (1991).

${ }^{11}$ G. Yu, C. Zhang, and A. J. Heeger, Appl. Phys. Lett. 64, 1540 (1994).

${ }^{12}$ J. C. Hummelen, B. W. Knight, F. LePeq, and F. Wudl, J. Org. Chem. 60, 532 (1995).

${ }^{13}$ K. Lee and H. Kim, Curr. Appl. Phys. 4, 323 (2004).

${ }^{14}$ H. Kim, J. S. Jee, J. Kim, H. Suh, and K. Lee (unpublished).

${ }^{15}$ C. Reber, L. Yee, J. McKiernan, J. I. Zink, R. S. Williams, W. M. Tong, D. A. A. Ohlberg, R. L. Whetlen, and F. Diederich, J. Phys. Chem. 95, 2127 (1991).

${ }^{16}$ M. Cocchi, D. Virgili, G. Giro, V. Fattori, P. DiMarco, J. Kalinowski, and Y. Shirota, Appl. Phys. Lett. 80, 2401 (2002).

${ }^{17}$ C. Y. Yang and A. J. Heeger, Synth. Met. 83, 85 (1996).

${ }^{18}$ J. K. J. van Duren, X. Yang, J. Loos, C. W. T. Bulle-Lieuwma, A. B. Sieval. J. C. Hummelen, and R. A. J. Janssen, Adv. Funct. Mater. 14, 425 (2004).

${ }^{19}$ G. Parthasarathy, C. Adachi, P. E. Burrows, and S. R. Forrest, Appl. Phys. Lett. 76, 2128 (2000).

${ }^{20}$ N. C. Greenham and P. A. Bobbert, Phys. Rev. B 68, 245301 (2003). 